\title{
Human-machine interfaces expand the functionality of prosthetic limbs
}

$\mathrm{N}$ eural interface technology is driving the development of increasingly sophisticated and practical prosthetic limbs, as illustrated by two papers recently published in Science Translational Medicine.

"Despite the advances in mechanotronics of prosthetic limbs, the ability to restore sensation was missing," explains Dustin Tyler, who led one of the studies. "Sensation is particularly challenging, because it requires a direct interface to the nerves."

Tyler and his colleagues at Case Western Reserve University (Cleveland, OH, USA) used peripheral nerve cuff electrodes to create a neural interface between a prosthetic hand and the residual limb in two upper limb amputees. When trains of electrical pulses were applied to the electrodes, touch sensations were generated, which were perceived by the patients as originating from the prosthetic hand.

By altering the pattern of the electrical stimulus, different sensations-including tapping, pressure and vibration - could be elicited. The amputees successfully used this sensory feedback to perform tasks that required fine motor coordination, such as pulling the stem from a cherry, or picking a grape from a bunch.

"We don't think that we introduce exactly the same information content as that of an intact hand," acknowledges Tyler. "But we believe it is close enough that the brain can interpret the information as a normal sensation." He compares this phenomenon to an optical illusion, in which the brain assembles inaccurate or incomplete information into a pattern that matches its previous experience.

The second study, led by Max OrtizCatalan at Chalmers University of Technology, Gothenburg, Sweden, highlights another drawback of standard prosthetic limbs. "The conventional attachment of limb prostheses requires a socket, which compresses the soft tissue at the stump to keep the prosthesis in place," explains Ortiz-Catalan. "This permanent insult on the skin and and soft tissue is known to be a source of several problems for amputees."

To address this issue, Rickard Brånemark and co-workers at Sahlgrenska University Hospital in Gothenburg developed an osseointegration approach, in which a prosthetic limb is directly and stably attached to the patient's skeleton. In collaboration with Brånemark, OrtizCatalan and colleagues combined this technology with an implanted neuromuscular interface to produce a myoelectrically controlled robotic hand that could readily be used outside the laboratory environment.

The device, termed an osseointegrated human-machine gateway (OHMG), was fitted to a patient with a trans-humeral amputation. "Our patient was chosen because he had several problems with his previous prosthesis, which would not work in cold environments, or when he raised his arm," explains Ortiz-Catalan. The researchers found that the patient could use the prosthetic hand not only to perform activities of daily living, but also to continue his physically demanding career as a truck driver.

"The most significant contribution of this work is that for the first time, amputee patients can actually benefit from neuromuscular interfaces," concludes Ortiz-Catalan. "Previously, they have been limited to participation in research in controlled environments, then leaving for home without such technologies."

The OHMG allows bidirectional communication to neuromuscular interfaces and, therefore, has the capacity to provide sensory feedback as well as volitional control. Like Tyler and colleagues, Ortiz-Catalan's team were able to simulate a sense of touch in the prosthetic hand by applying electrical stimulation to the OHMG electrodes.

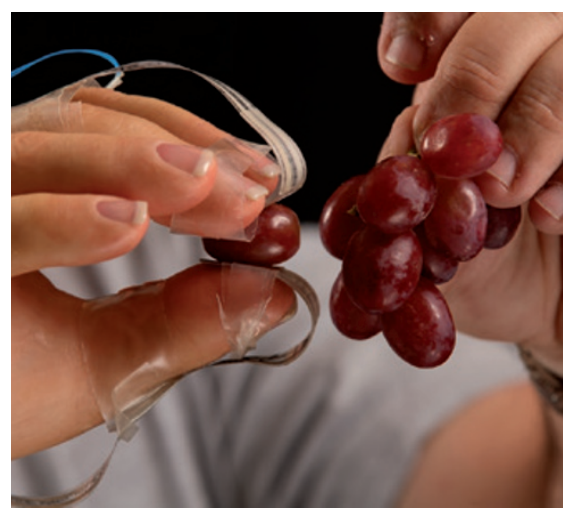

Artificial touch sensation in a prosthetic hand enables an upper limb amputee to perform fine motor movements, such as pulling a grape from a bunch. Image courtesy of D. Omori.

So far, this functionality has only been tested in the laboratory, and remains to be explored in real-world settings.

Ortiz-Catalan plans to take advantage of the portability of the OHMG to further develop its capabilities. "We have the unique opportunity to chronically record and stimulate the neuromuscular system in patients performing their daily life routines," he says. “Therefore, we will continue to explore neurostimulation to provide somatosensory perception, as well as neuromuscular decoding of motor control."

The device designed by Tyler's team currently relies on percutaneous leads to deliver the electrical stimulation, but the researchers are now working towards a fully implantable system. "We have found that sensation provides the patient with the sense of having their hand back," Tyler concludes. "When the patient leaves the laboratory, he comments that he is sad that he has to leave his hand behind."

\section{Heather Wood}

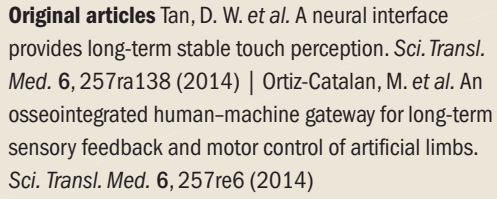
provides long-term stable touch perception. Sci. Transl. Med. 6, 257ra138 (2014) | Ortiz-Catalan, M. et al. An osseointegrated human-machine gateway for long-term sensory feedback and motor control of artificial limbs. Sci. Transl. Med. 6, 257re6 (2014) 